\title{
73.7 Tb/s (96X3×256-Gb/s) mode-division-multiplexed DP-16QAM transmission with inline MM-EDFA
}

\author{
V.A.J.M. Sleiffer ${ }^{(1)}$, Y. Jung ${ }^{(2)}$, V. Veljanovski( ${ }^{(3)}$, R.G.H. van Uden ${ }^{(1)}$, M. Kuschnerov ${ }^{(3)}$, Q. Kang ${ }^{(2)}$, \\ L. Grüner-Nielsen ${ }^{(4)}$, Y. Sun ${ }^{(4)}$, D.J. Richardson ${ }^{(2)}$, S. Alam ${ }^{(2)}$, F. Poletti ${ }^{(2)}$ J.K. Sahu ${ }^{(2)}$, A. Dhar ${ }^{(2)}$ ', \\ H. Chen ${ }^{(1)}$, B. Inan ${ }^{(5)}$, A.M.J. Koonen ${ }^{(1)}$, B. Corbett ${ }^{(6)}$, R. Winfield ${ }^{(6)}$, A.D. Ellis ${ }^{(6)}$, and H. de Waardt ${ }^{(1)}$ \\ (1) COBRA institute, Eindhoven University of Technology, The Netherlands, v.a.j.m.sleiffer@tue.nl \\ (2) Optoelectronics Research Centre, University of Southampton, Southampton, SO17 1BJ, UK \\ (3) Nokia Siemens Networks GmbH \& Co. KG, Munich, Germany \\ (4) OFS, Priorparken 680, 2605 Brøndby, Denmark \\ (5) Technische Universität München, Munich, Germany \\ (6) Tyndall National Institute, Cork, Ireland
}

Abstract We show transmission of a $73.7 \mathrm{~Tb} / \mathrm{s} \quad(96 \times 3 \times 256-\mathrm{Gb} / \mathrm{s})$ DP-16QAM modedivision-multiplexed signal over $119 \mathrm{~km}$ of few-mode fiber with inline multi-mode EDFA, using $6 \times 6$ MIMO digital signal processing. The total demonstrated net capacity is $57.6 \mathrm{~Tb} / \mathrm{s}$ (SE $12 \mathrm{bits} / \mathrm{s} / \mathrm{Hz}$ ).

\section{Introduction}

To avoid, or at least delay, the upcoming capacity crunch, research is looking into space division multiplexing (SDM) as the most viable way to increase the capacity per fiber by several orders of magnitude. Research groups around the world are focusing on two primary SDM media: The multi-core fiber $^{1-3}$ and multimode fiber $^{4-11}$. From an energy perspective, the latter has been shown to be more efficient when it comes to amplification [12] and it potentially offers a higher information capacity flow per unit area, making it a very interesting proposition.

Using a few-mode fiber (FMF) which supports three spatial modes and MIMO processing, the authors in [6] showed for the first time mode-division-multiplexed (MDM) transmission was feasible. Since then the transmission distance was improved using costly Raman amplification and a low-loss spatial (de-)multiplexer. However, always a single span and a single or low number of wavelengths was used ${ }^{7}$. A $1200 \mathrm{~km}$ distance was obtained going to the single-mode regime after each re-circulating loop $^{8}$, which would be impractical for long-haul transmission. In [9] the first wavelength-division multiplexed (WDM) MDM transmission was shown using 88 wavelengths carrying $112-\mathrm{Gb} / \mathrm{s}$ dual-polarization (DP)-QPSK over $50 \mathrm{~km}$ of FMF. A larger distance $(85 \mathrm{~km})$ was shown using a MM-EDFA mid-span ${ }^{10}$, but using only a single wavelength.

In this work, we present the first WDM FMF experiment with a mid-span amplifier, successfully transmitting 96 WDM channels over $119 \mathrm{~km}$, using $256-\mathrm{Gb} / \mathrm{s}$ DP-16QAM modulation. We demonstrate $73.7-\mathrm{Tb} / \mathrm{s}$ gross data rate transmission with a net spectral efficiency (SE) of $12 \mathrm{bits} / \mathrm{s} / \mathrm{Hz}$ (assuming 20\% soft-decision forward-error correction code (SD-FEC)). To the best of our knowledge this is the highest capacity so far reported for MDM transmission.

\section{Experimental Setup}

Fig. 1 depicts the experimental setup. At the transmitter side, 96 channels carrying a

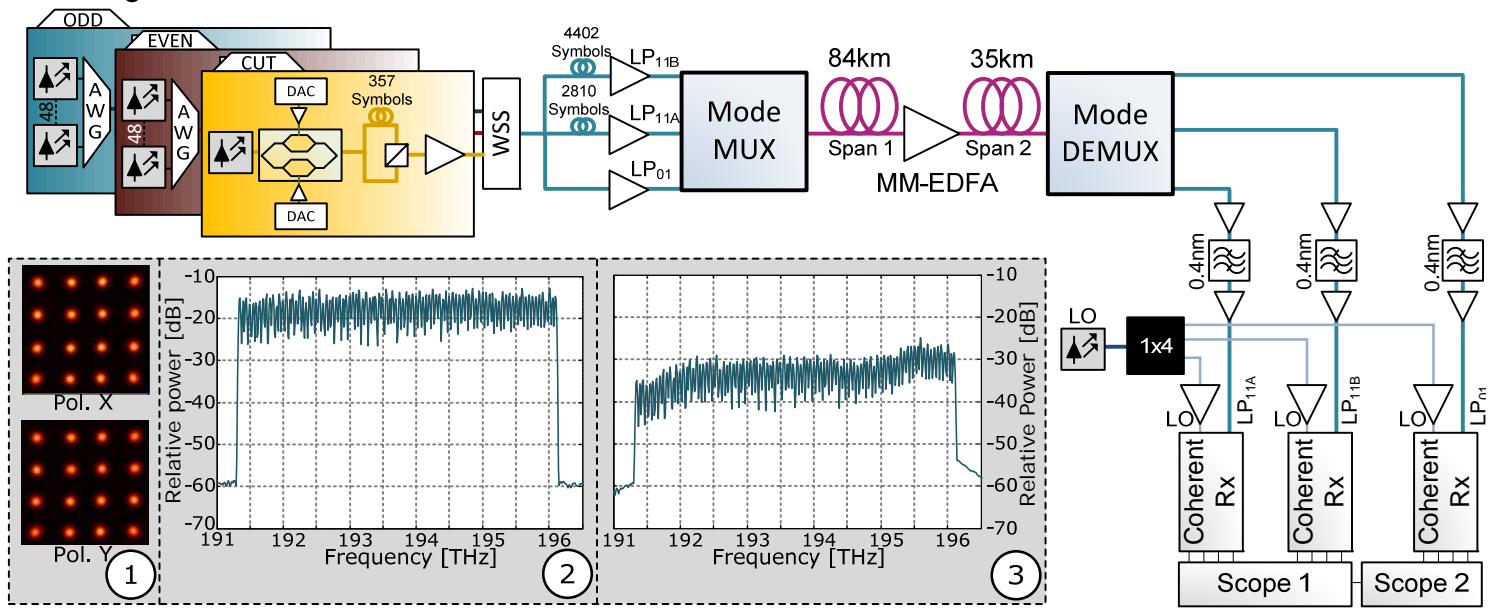

Fig. 1. Experimental setup. 1: 256-Gb/s DP-16QAM Constellations back-to-back single-mode. 2: Spectrum at transmitter side (96 channels). 3: Spectrum after DEMUX 
256-Gb/s DP-16QAM modulated signal were created. The channels are composed of 48 even and 48 odd channels, of which one is dropped and a channel under test (CUT) is inserted. The even and odd channels were generated by multiplexing $48 \mathrm{ECL}$ lasers running on the 50-GHz ITU-grid (191.35 THz - 196.1 THz), using AWGs. Subsequently the signals containing 48 wavelengths each are $128-\mathrm{Gb} / \mathrm{s}$ 16QAM modulated using IQ-modulators. The modulators are driven with electrical 4-PAM signals, created by separate DACs for the inphase (I) and quadrature (Q) port. These 4PAMs were created in the digital domain by adding two PRBS 13 sequences together, shifted by 383 symbols for de-correlation. The I and $Q$ were shifted with respect to each other by 767 symbols. The outputs of the DACs were electrically amplified before feeding them to the IQ-modulators. The output swing was set such that the IQ-modulators were operated in the linear regime, no pre-distortion was applied. After modulation the 256-Gb/s DP-16QAM signals were emulated by polarization-multiplexing (POLMUX) the outputs of the IQ-modulators. This was achieved by splitting the signal into two equally powered tributaries, delaying one by $\sim 200$ symbols, and combining them again using a polarization-beam combiner. The CUT was generated the same way as the even and odd channels, with the exception that only one laser was modulated and different sequences and delays were used. The 4-PAMs were generated using PRBS 15 sequences, shifted by 8191 symbols. Between the I and Q a 16383 symbol shift was applied. In the POLMUX stage of the CUT one of the tributaries was delayed by 357 symbols with respect to the other. Finally, the three setups are multiplexed using a wavelength-selective switch (WSS), and equalized. One of the even or odd channels was dropped and the CUT was inserted on that wavelength instead. This gives us the opportunity to freely tune the CUT over the spectrum, without the need to tune the offset between the carrier wavelength and local oscillator (LO) for every channel.

After multiplexing the signal containing 96x256-Gb/s DP-16QAM is split up into three equally powered signals, which are fed to the phase-plate based mode multiplexer (MUX) built as reported in [11]. The signals going to the $\mathrm{LP}_{11 \mathrm{a}}$ port and $\mathrm{LP}_{11 \mathrm{~b}}$ port have delays of 2810 and 4402 symbols, respectively, with respect to the signal going to the $\mathrm{LP}_{01}$ port.

The transmission link consisted of $\mathrm{FMF}^{13}$ and a MM-EDFA ${ }^{14}$. The first span consisted of three FMF spools with lengths of $30 \mathrm{~km}, 30 \mathrm{~km}$ and $24 \mathrm{~km}$. The second span was built up out of one $30 \mathrm{~km}$ and one $5 \mathrm{~km}$ spool. The fiber characteristics are listed in Tab. 1. The spools in the first span were combined such that the average differential mode delay (DMD) over the span was around 15 symbols (457.4 ps). The MM-EDFA after the first span provided $\sim 18 \mathrm{~dB}$ of gain per mode.

After the transmission link the signal was sent into a mode de-multiplexer (DEMUX) which again is built as reported in [11]. The outputs of the DEMUX, were amplified using SM-EDFAs. After amplification the CUT was filtered out using a $50 \mathrm{GHz}$ tunable optical filter. After another amplification stage, the signal was sent into commercial coherent receivers using single-ended detection with trans-impedance amplifiers. The $\mathrm{LP}_{11 \mathrm{a}}$ and $\mathrm{LP}_{11 \mathrm{~b}}$ receivers were connected to the same $40 \mathrm{GSamples} / \mathrm{s}$ digital sampling scope (scope 1 in Fig. 1), whereas the $\mathrm{LP}_{01}$ receiver was connected to a 50 GSamples/s digital sampling scope (scope 2 ). The delays between the scopes and signals were carefully synchronized before measuring. The samples obtained from the scopes were processed offline using data-aided DSP. 800,000 from scope 1 and 1,000,000 samples from scope 2 were captured, resulting in 640,000 DP-16QAM symbols per mode. First the chromatic dispersion was blindly estimated on the $\mathrm{LP}_{01}$ received signal and compensated for subsequently on all received modes. After applying feed-forward timing recovery, a time-domain 6x6 MIMO equalizer (TDE), containing 401 taps (200 symbols), 260,000 symbols were used for decision-directed LMS for convergence. This leaves 1.5 Million bits per spatial-polarization mode for bit-error rate (BER) evaluation. Per measured BER point at least two shots at different time instances are processed and averaged.

Tab. 1: Span 1 (spool 1,2,3) (total length 84km) and span 2 (spool 4,5) (total length 35km)

\begin{tabular}{|c|c|c|c|c|c|}
\cline { 2 - 6 } & Spool 1 & Spool 2 & Spool 3 & Spool 4 & Spool 5 \\
\hline Length $[\mathrm{m}]$ & 30000 & 29980 & 23809 & 29980 & 5030 \\
\hline MPI [dB] & -26 & -26 & -26 & -26 & Unknown \\
\hline DMD [ps/m] & 0.060 & -0.034 & -0.052 & 0.039 & -0.006 \\
\hline Dispersion LP01 $[\mathrm{ps} /(\mathrm{nm} \cdot \mathrm{km})]$ & 19.9 & 19.8 & 19.8 & 20.1 & 19.8 \\
\hline Dispersion LP $11[\mathrm{ps} /(\mathrm{nm} \cdot \mathrm{km})]$ & 20.0 & 20.0 & 19.9 & 20.0 & 19.9 \\
\hline
\end{tabular}




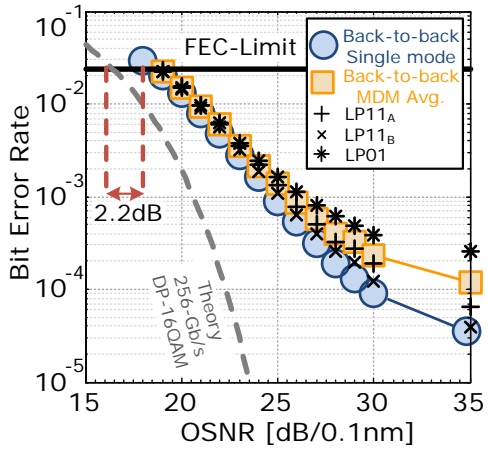

Fig. 2. Back-to-back curves.

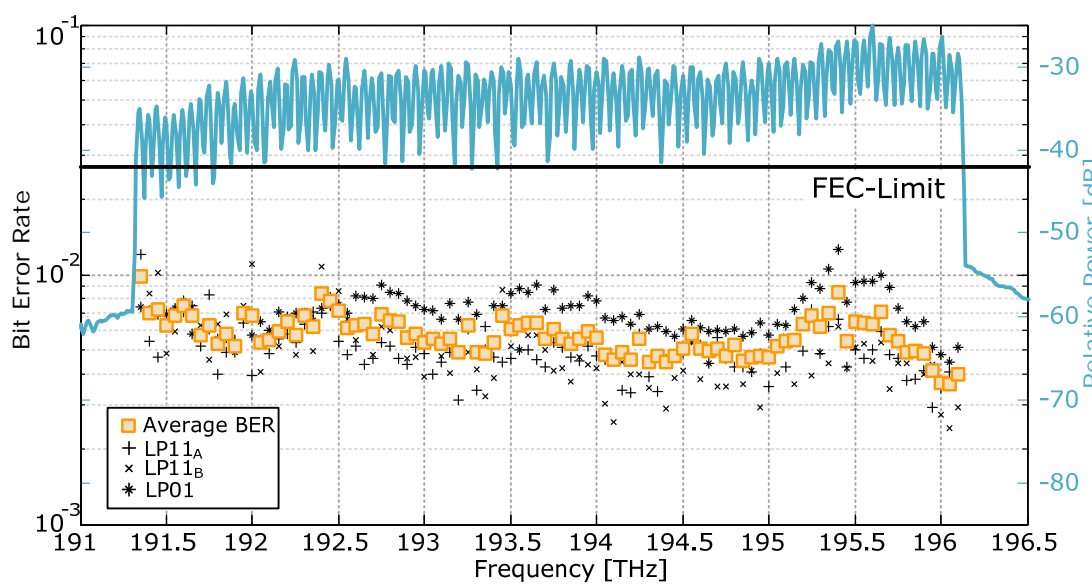

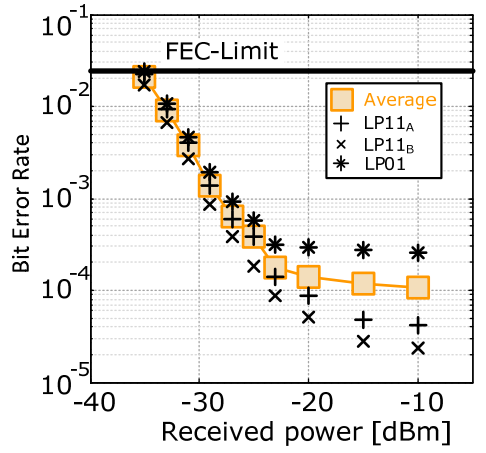

Fig. 3. Bit error rate vs. received power per channel after DEMUX back-to-back

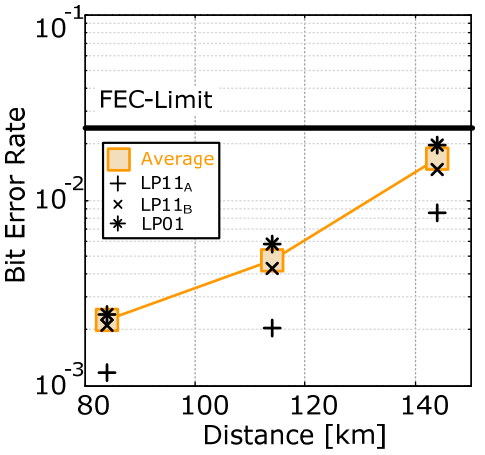

Fig. 4. Bit error rate vs. distance for the 193.40THz channel

Fig. 5. Bit error rate for all 96 DWDM channels after $119 \mathrm{~km}$ transmission distance. Right: Constellations after transmission.

\section{Results}

Fig. 2 depicts the OSNR vs. BER curves measured back-to-back in single mode and back-to-back using the mode MUX and DEMUX. An SD-FEC ${ }^{15}$ overhead of $20 \%$ was assumed which translates into a FEC-limit at a BER of $2.4 \cdot 10^{-2}$. The single mode results show a $2.2 \mathrm{~dB}$ OSNR-penalty at the FEC-limit with respect to theory. An error floor of $2 \cdot 10^{-5}$ is observed. For the MDM setup there is no additional penalty at the FEC-limit compared to single mode. For this setup an error-floor at $1 \cdot 10^{-4}$ is observed, which is mainly caused by the LP01 performance.

Fig. 3 shows the output power out of the DEMUX per spatial-mode per wavelength channel, versus BER in a back-to-back configuration. This plot shows that ultimately the output power limits the performance due to ASE produced in the SM-EDFAs at the receiver side. Fig. 4 shows the transmission distance versus BER. With keeping a decent margin with respect to the FEC-limit, we decided to transmit over $119 \mathrm{~km}$ of fiber.

Fig. 5 shows the transmission results for all the $963 \times 256-G \mathrm{~b} / \mathrm{s}$ DP-16QAM modulated channels over $119 \mathrm{~km}$ of few-mode fiber. All WDM channels performed well below the FEC-limit for each separate mode.

\section{Conclusions}

We successfully transmitted $73.7 \mathrm{~Tb} / \mathrm{s}$ (96x3×256-Gb/s) mode-division-multiplexed DP-16QAM over $119 \mathrm{~km}$ of few-mode fiber, demonstrating a total system net capacity of 57.6 Tb/s. Substantial further increases in transmission distance should be possible by reduction of the mode coupling losses.

This work was supported by the EU FP7-ICT MODE-GAP project under grant agreement 228033.

\section{References}

[1] S. Chandrasekhar et al., Proc. ECOC'11, Th.13.C.4

[2] R. Ryf et al., Proc. OFC'12, PDP5C.2

[3] J. Sakaguchi et al., Proc OFC'12, PDP5C.1

[4] A. Li et al., Proc. OFC'11, PDPB8

[5] C. Koebele et al., Proc, ECOC'11, Th.13.C.3

[6] R. Ryf et al., Proc. OFC'11, PDPB10

[7] R. Ryf et al., Proc. Sum. Top. '12, MC3.2

[8] S. Randel et al. Proc. OFC'12, PDP5C.5

[9] E. Ip et. al., Proc. ECOC'11, Th.13.C.2

[10] E. Ip et al., Proc. OFC'12, Otu2C.4

[11] V.Sleiffer et al., Proc. ECOC'11, TU.1.C.2

[12] P.M. Krummrich et al., Proc. OFC'12, OMH5

[13] L. Grüner-Nielsen et al., Proc. OFC'12, PDP5A.1

[14] Y. Jung et al., Opt. Exp. 19, B952 (2011)

[15] D.A. Morero et. al, Globecom (2011) 